\title{
The LF/HF ratio does not accurately measure cardiac sympatho-vagal balance
}

\section{George E. Billman*}

Department of Physiology and Cell Biology, The Ohio State University, Columbus, OH, USA

*Correspondence: billman.1@osu.edu

\section{Edited by:}

Jerzy Sacha, Regional Medical Center, Poland

Reviewed by:

Jerzy Sacha, Regional Medical Center, Poland

Power spectral analysis of the beat-tobeat variations of heart rate or the heart period ( $\mathrm{R}-\mathrm{R}$ interval) has become widely used to quantify cardiac autonomic regulation (Appel et al., 1989; Task Force of the European Society of Cardiology and the North American Society of Pacing and Electrophysiology, 1996; Berntson et al., 1997; Denver et al., 2007; Thayler et al., 2010; Billman, 2011). This technique partitions the total variance (the "power") of a continuous series of beats into its frequency components, typically identifying two or three main peaks: Very Low Frequency (VLF) $<0.04 \mathrm{~Hz}$, Low Frequency (LF), $0.04-0.15 \mathrm{~Hz}$, and High Frequency (HF) $0.15-0.4 \mathrm{~Hz}$. It should be noted that the HF peak is shifted to a higher range (typically $0.24-1.04 \mathrm{~Hz}$ ) in infants and during exercise (Berntson et al., 1997). The HF peak is widely believed to reflect cardiac parasympathetic nerve activity while the LF, although more complex, is often assumed to have a dominant sympathetic component (Task Force of the European Society of Cardiology and the North American Society of Pacing and Electrophysiology, 1996; Berntson et al., 1997; Billman, 2011). Based upon these assumptions, Pagani and co-workers proposed that the ratio of LF to HF (LF/HF) could be used to quantify the changing relationship between sympathetic and parasympathetic nerve activities (i.e., the sympatho-vagal balance) (Pagani et al., 1984, 1986; Malliani et al., 1991) in both health and disease. However, this concept has been challenged (Kingwell et al., 1994; Koh et al., 1994; Hopf et al., 1995; Eckberg, 1997; Houle and Billman, 1999; Billman, 2011). Despite serious and largely under-appreciated limitations, the LF/HF ratio has gained wide acceptance as a tool to assess cardiovascular autonomic regulation where increases in LF/HF are assumed to reflect a shift to "sympathetic dominance" and decreases in this index correspond to a "parasympathetic dominance." Therefore, it is vital to provide a critical assessment of the assumptions upon which this concept is based.

The hypothesis that LF/HF accurately reflects sympatho-vagal balance rests upon several interrelated assumptions as follows (modified from Eckberg, 1997): (1) cardiac sympathetic nerve activity is a major, if not the exclusive, factor responsible for the LF peak of the heart rate power spectrum; (2) cardiac parasympathetic is exclusively responsible for the HF peak of the heart rate power spectrum; (3) disease or physiological challenges provoke reciprocal changes in cardiac sympathetic and parasympathetic nerve activity (i.e., increases in cardiac parasympathetic nerve activity are always accompanied with corresponding reductions in cardiac sympathetic nerve activity and vice versa); and (4) there is a simple linear interaction between the effects of cardiac sympathetic and cardiac parasympathetic nerve activity on heart rate variability (HRV).

As previously noted, frequency domain analysis of HRV usually reveals two or more peaks, a lower frequency $(<015 \mathrm{~Hz})$ and a higher frequency peak $(>0.15 \mathrm{~Hz})$ that are often assumed to correspond to cardiac sympathetic and cardiac parasympathetic neural activity, respectively (Pagani et al., 1984, 1986; Malliani et al., 1991). However, accumulating evidence clearly demonstrates that this assumption is naive and greatly oversimplifies the complex non-linear interactions between the sympathetic and the parasympathetic divisions of the autonomic nervous system (Berntson et al., 1997; Eckberg, 1997; Parati et al., 2006; Billman, 2009, 2011). This is particularly true with regards to the relationship between LF power and cardiac sympathetic regulation (Randall et al., 1991; Ahmed et al., 1994; Kingwell et al., 1994; Hopf et al., 1995; Eckberg, 1997; Houle and Billman, 1999; Parati et al., 2006; Billman, 2009, 2011).

The LF peak of the heart rate power spectrum is reduced by at least $50 \%$ by either cholinergic antagonists or selective parasympathectomy (Akselrod et al., 1981; Randall et al., 1991; Houle and Billman, 1999). Importantly, this peak is not completely eliminated by the combination of selective denervation and betaadrenoceptor blockade (Randall et al., 1991); 25\% of the peak remains after this treatment. As a consequence, LF/HF often actually increases from baseline values when both parasympathetic and adrenergic nerve activity have been blocked. For example, using the data reported by Randall and co-workers (Randall et al., 1991), LF/HF increased from a baseline value of 1.1-8.4 when selective parasympathetic denervation was combined with beta-adrenergic receptor blockade, falsely suggesting a major shift to sympathetic dominance! In a similar manner, interventions that would be expected to increase cardiac sympathetic activity, such as acute exercise or myocardial ischemia, not only failed to increase LF power but actually provoked significant reductions in this variable (Houle and Billman, 1999), once again yielding LF/HF values that are difficult to interpret. Indeed, despite large increases in heart rate, LF/HF ratio was largely unaffected by either acute myocardial ischemia, exercise, or the cholinergic antagonist atropine sulfate (Houle and Billman, 1999). Finally, direct recording of sympathetic nerve activity failed to correlate with LF power in either healthy subjects or patients with heart failure (Hopf et al., 1995; Notarius and Floras, 2001; Jardine et al., 2002; Moak et al., 
2007; Piccirillo et al., 2009), a condition known to increase cardiac sympathetic drive (Hasking et al., 1986; Saul et al., 1988; Watson et al., 2007). Thus, the LF component of HRV does not provide an index of cardiac sympathetic drive but rather reflects a complex and not easily discernible mix of sympathetic, parasympathetic, and other unidentified factors with parasympathetic factors accounting for the largest portion of the variability in this frequency range. As a consequence, the physiological basis for $\mathrm{LF} / \mathrm{HF}$ is difficult to discern.

Although the vast majority of the clinical and the experimental studies demonstrate a strong association between HF power and cardiac parasympathetic activity (Katona et al., 1970; Appel et al., 1989; Billman and Hoskins, 1989; Billman and Dujardin, 1990; Task Force of the European Society of Cardiology and the North American Society of Pacing and Electrophysiology, 1996; Billman, 2009, 2011; Thayler et al., 2010), this concept has also been challenged (Kollai and Mizsei, 1990; Goldberger et al., 1994; Hedman et al., 1995; Taylor et al., 2001; Parati et al., 2006). Unlike LF power and sympathetic nerve activity, a strong correlation between HF power and direct recordings of cardiac parasympathetic activity has been reported (Chess et al., 1975; Piccirillo et al., 2009). However, just as parasympathetic activation exerts profound influences on the LF component of HRV, sympathetic neural activity may modulate the HF component of the $\mathrm{R}-\mathrm{R}$ interval variability (Taylor et al., 2001; Cohen and Taylor, 2002). Taylor et al. (2001) found that cardioselective beta-adrenergic receptor blockade (drugs that should not indirectly alter vagal outflow via action within the central nervous system) increased the amplitude of the respiratory sinus arrhythmia over a wide range of respiratory frequencies (i.e., the increases were not restricted to lower frequencies, $<0.15 \mathrm{~Hz}$ ). They concluded that "cardiac sympathetic outflow can oppose vagally mediated $R-R$ interval oscillations and sympathetic blockade removes this effect" (Cohen and Taylor, 2002). Based upon these data, sympathetic nerve activation may alter the HF peak by perhaps as much as $10 \%$. Thus, differences in cardiac sympathetic activation during a physiological challenge (e.g., exercise or postural changes) in healthy subjects or that occur as the consequence of cardiovascular disease (following myocardial infarction) could restrain vagally mediated changes in HRV. These data suggest that HF power cannot be solely attributed to changes in cardiac vagal efferent nerve traffic, further compromising an accurate interpretation of the $\mathrm{LF} / \mathrm{HF}$ ratio.

Accurate interpretation of LF/HF ratio also depends upon the assumption that physiological interventions always elicit reciprocal changes in parasympathetic and sympathetic nerve activity. However, following the termination of exercise sympathetic activation remains high despite the rapid re-activation of cardiac parasympathetic drive (Smith et al., 2005; Billman and Kukielka, 2007; Billman, 2009). Furthermore, chemoreceptor activation by carbon dioxide provokes parallel reductions in sympathetic and parasympathetic nerve activity (Eckberg, 1997) while facial emersion in cold water (activating the so-called "diving reflex") increased sympathetic nerve activity yet elicited a profound bradycardia (Eckberg et al., 1984; Fagius and Sundlof, 1986). The observation that heart rate declines, despite increases in sympathetic nerve activity, highlights the complex non-linear interactions of the sympathetic and parasympathetic nervous system, providing an example of "accentuated antagonism" (Levy, 1971; Stramba-Badiale et al., 1991; Uijtdehaage and Thayer, 2000), the dominance of parasympathetic over sympathetic influences on cardiac rate. Finally, reciprocal changes in parasympathetic and sympathetic nerve activity do not always occur even during the activation of the baroreceptor reflex (Eckberg, 1997). Eckberg and co-workers have shown that, although small changes in arterial pressure typically provoke reciprocal changes sympathetic and parasympathetic nerve activity, large increases in arterial pressure only provoke increases in parasympathetic nerve activity without altering the prevailing sympathetic activity (Eckberg, 1980; Rea and Eckberg, 1987). Furthermore, autonomic response to baroreceptor reflex activation depends on whether the pressure changes occur near the threshold or the saturation point of the response curve; the same change in pressure can elicit larger or smaller autonomic responses depending on how close the prevailing pressure lies to the threshold (larger) or saturation (smaller) portion of the stimulus-response curve (Eckberg, 1980, 1997). As previously noted, changes in heart rate do not result from the simple algebraic summation of the sympathetic and parasympathetic nerve activity. Rather, parasympathetic nerve activation can completely override even maximal sympathetic nerve stimulation, provoking large reductions in heart rate in the face of sympathetic nerve activation as was previously noted for the diving reflex. Thus, physiological interventions can elicit either complex non-linear reciprocal or parallel changes in either division of the autonomic nervous system. These complex interactions can profoundly influence the calculation and the interpretation of LF/HF.

Mathematical considerations can also influence LF/HF values. Similar LF/HF values can be obtained via either exclusive changes in the numerator (i.e., LF), or the dominator (i.e., HF), or by some combination of the two, as is illustrated in Table 1. For example, a doubling of parasympathetic activity against maintained sympathetic nerve activation yields the identical LF/HF value as a 50\% reduction in sympathetic nerve activity against a constant background parasympathetic regulation. Based upon the literature, one can conclude that parasympathetic nerve activation contributes to at least $50 \%$ of the LF variability while sympathetic activity, at best, only contributes $25 \%$ to this variability (Randall et al., 1991). A substantial portion of the variability in the LF band also results from other unidentified factors. In a similar fashion, sympathetic nerve activity could contribute to perhaps as much as $10 \%$ of the $\mathrm{HF}$ variability (Taylor et al., 2001; Cohen and Taylor, 2002). As a consequence, the effects of changing sympathetic and parasympathetic activity on the LF/HF are quite variable and not intuitively obvious, as is illustrated in Figure 1. This figure was constructed using the following formula that was based upon a synthesis of the literature (particularly, Randall et al., 1991; Taylor et al., 2001; Cohen and Taylor, 2002), LF $=0.5$ parasympathetic +0.25 sympathetic activity while $\mathrm{HF}=0.9$ parasympathetic +0.1 sympathetic nerve activity. The nerve activity was varied from baseline ( 1 arbitrary unit each) increasing or decreasing by up to a 
Table 1 | Examples of the effects of varying cardiac sympathetic and parasympathetic nerve activity on LF/HF.

\begin{tabular}{lllll}
\hline $\begin{array}{l}\text { Parasympathetic nerve } \\
\text { activity }\end{array}$ & $\begin{array}{l}\text { Sympathetic nerve } \\
\text { activity }\end{array}$ & LF & HF & LF/HF \\
\hline 1 & 1 & 0.75 & 1 & 0.75 \\
2 & 1 & 1.25 & 1.9 & 0.66 \\
0.5 & 1 & 0.5 & 0.55 & 0.91 \\
1 & 2 & 1 & 1.1 & 0.91 \\
1 & 0.5 & 0.625 & 0.95 & 0.66 \\
2 & 2 & 1.5 & 2 & 0.75 \\
2 & 0.5 & 1.125 & 1.85 & 0.61 \\
0.5 & 2 & 0.75 & 0.65 & 1.15 \\
0.5 & 0.5 & 0.375 & 0.5 & 0.75 \\
\hline
\end{tabular}

These numbers were generated using the following formula (derived from a synthesis of the literature, particularly Randall et al., 1991; Taylor et al., 2001; Cohen and Taylor, 2002): LF/HF $=10.5$ parasympathetic + 0.25 sympathetic nerve activity)/(0.9 parasympathetic +0.1 sympathetic nerve activity). The nerve activity is reported as arbitrary units where at baseline sympathetic and parasympathetic nerve activity were normalized as 1 arbitrary unit each. The data shown are for baseline and various combinations of doubling $(2 \times$ baseline) or halving (0.5 $\times$ baseline) the autonomic nerve activity.

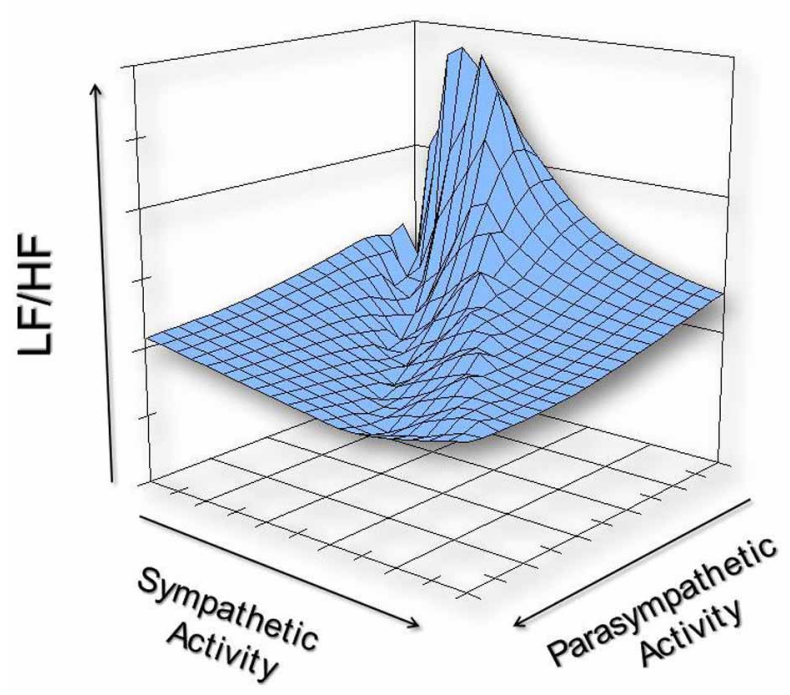

FIGURE 1 | An illustration of the possible non-linear effects of varying cardiac sympathetic and cardiac parasympathetic nerve activity on LF/HF. This graph was constructed using the following formula (derived from a synthesis of the literature, particularly Randall et al., 1991; Taylor et al., 2001; Cohen and Taylor, 2002): LF/HF $=(0.5$ parasympathetic +0.25 sympathetic nerve activity)/(0.9 parasympathetic +0.1 sympathetic nerve activity). The nerve activity was varied from baseline (1 arbitrary unit each) increasing or decreasing by up to a factor of 10 (i.e., from 0.1 to 10 units).

factor of 10 . Due to the substantial contribution (accounting for up to $25 \%$ of the variability) (Randall et al., 1991) from non-neural factors to LF power, very distorted values of LF/HF can be obtained when both sympathetic and parasympathetic nerve activity are minimal. If for example, one assumes that $\mathrm{LF}=0.5$ parasympathetic +0.25 sympathetic + 0.25 other factors and both parasympathetic and sympathetic nerve activity are reduced to $1 / 100$ the baseline values, the calculated LF/HF becomes $(0.005+$ $0.0025+0.25) /(0.009+0.001)=25.75$ ! Despite the almost complete absence of cardiac autonomic regulation, this value could be inappropriately interpreted as a major shift toward sympathetic dominance. Furthermore, LF/HF cannot be determined if both sympathetic activity and parasympathetic nerve activity were to be abolished completely (i.e., when the dominator is zero). Finally, mathematical complications also arise due to the nonlinear relationship between $\mathrm{R}-\mathrm{R}$ interval and heart rate; similar changes in heart rate elicit much greater variability in $\mathrm{R}$ $\mathrm{R}$ interval at lower than at higher heart rates (Sacha and Pluta, 2008). As a consequence of this non-linear relationship, it is difficult to separate the changes in HRV that arise from direct action of cardiac autonomic nerves from those changes that result indirectly from neurally induced changes in average heart rate. This observation led Sacha and Pluta (2008), to propose that HRV has both physiological and mathematical influences that can be corrected by the division of HRV by average $\mathrm{R}-\mathrm{R}$ interval. Thus, the physiological basis for changes in LF/HF is not readily discernible and spurious values for $\mathrm{LF} / \mathrm{HF}$ can result as a consequence of the mathematical manipulations of the data.

It should also be noted, that HRV (and thereby LF/HF) is affected by respiratory parameters and mechanical events independent of changes in cardiac autonomic nerve activity. The contribution of mechanical factors (due to stretch of the atria that results from both changes in cardiac filling and the changing thoracic pressure that occur during respiration) to changes in HRV was first proposed by Bainbridge (1930). This conclusion is supported by the observation that heart transplant patients, despite the absence of cardiac nerves, still exhibit small ( $\sim 2-8 \%$ of normal) change in $\mathrm{R}-\mathrm{R}$ interval associated with the respiratory cycle (Bernardi et al., 1989). Taylor et al. (2001) further demonstrated that atrial stretch can exert significant influences on $\mathrm{R}-\mathrm{R}$ interval in subjects with complete autonomic blockade. They found that after combined cholinergic and adrenergic receptor blockade slow deep breathing could still provoke oscillations of $\sim 120 \mathrm{~ms}$ in healthy human subjects (Taylor et al., 2001). In similar manner, mechanical distortion (stretch) of the sinoatrial nodal stretch in pigs without functional autonomic innervation (vagal nerve section combined with propranolol 
treatment) reduced the HF component of HRV (Horner et al., 1996).

Respiratory parameters can also profoundly alter heart rate and R-R interval variability independent of changes in cardiac autonomic regulation (i.e., against a constant background level of automatic regulation) (Angelone and Coulter, 1964; Davies and Neilson, 1967; Hainsworth, 1974; Melcher, 1976; Hirsch and Bishop, 1981; Brown et al., 1993; Van De Borne et al., 2001). It is now well established that increases in respiratory frequency reduce the amplitude of heart rate oscillations (Angelone and Coulter, 1964; Melcher, 1976; Hirsch and Bishop, 1981; Brown et al., 1993) while either increases in tidal (Davies and Neilson, 1967; Melcher, 1976; Hirsch and Bishop, 1981; Eckberg, 1983; Kollai and Mizsei, 1990; Brown et al., 1993) or static lung volume (Hainsworth, 1974) provoke increases in the $\mathrm{R}-\mathrm{R}$ interval variability. The facts are in direct opposiiton to the assumptions. Conversely, reductions in respiratory frequency increase HRV (Angelone and Coulter, 1964; Melcher, 1976; Hirsch and Bishop, 1981; Brown et al., 1993) while decreases in tidal volume lead to reductions in the $\mathrm{R}-\mathrm{R}$ interval variability (Davies and Neilson, 1967; Melcher, 1976; Hirsch and Bishop, 1981; Eckberg, 1983; Kollai and Mizsei, 1990; Brown et al., 1993). Thus, it is critical to control breathing (paced or timed breathing) in order to interpret HRV data accurately. For obvious reasons, it is much more difficult to control respiratory parameters in conscious animal than in human studies. However, these respiratory parameters frequently are not controlled even in human studies (Brown et al., 1993). Brown and co-workers (Brown et al., 1993), reviewed the human literature and found that only about $51 \%$ controlled respiratory rate, and even fewer studies controlled for tidal volume (11\%). They further reported that respiratory parameters not only altered HF power but also strongly influenced the LF components of the R-R interval power spectrum, a component that previously was viewed to vary independently of changes in respiration (Brown et al., 1993).

Finally, prevailing heart rate can also influence HRV. There are a number of studies that report a strong positive correlation between mean $\mathrm{R}-\mathrm{R}$ interval and various time domain indices of HRV (e.g., the standard deviation of normal beats, SDNN) (Kleiger et al., 1987; Van Hoogenhuyze et al., 1991; Fleiss et al., 1992) such that HRV was greater during longer mean $\mathrm{R}-\mathrm{R}$ intervals (slower heart rates) than at shorter mean $\mathrm{R}-\mathrm{R}$ intervals (faster heart rates). Frequency domain analysis of HRV is similarly affected by mean heart rate. Sacha and Pluta (2005) found that LF was directly related, while HF was indirectly related, to the average heart rate of the subject. As a consequence, they further report that LF/HF varied depending on heart rate, lower at slower and higher at faster heart rates. Thus, heart rate per se can influence LF/HF independent of changes cardiac autonomic nerve activity.

As we have seen, the hypothesis that LF/HF quantifies "sympatho-vagal balance" depends upon four interrelated assumptions, all of which can be proven to be false. The facts are in direct opposition to the assumptions. In particular, the complex nature of LF power, its exceedingly poor relationship to sympathetic nerve activation, and the non-linear (and often non-reciprocal) interactions between sympathetic and parasympathetic nerve activity that are confounded by the mechanical effects of respiration and prevailing heart rate, make it impossible to delineate the physiological basis for LF/HF with any degree of certainty. Thus, the LF/HF sympatho-vagal balance hypothesis has been disproven - the preponderance of evidence confirms that LF/HF data cannot accurately quantify cardiac "sympatho-vagal balance" either in health or disease.

\section{REFERENCES}

Ahmed, M. W., Kadish, A. H., Parker, M. A., and Goldberger, J. J. (1994). Effect of physiologic and pharmacologic adrenergic stimulation on heart rate variability. J. Am. Coll. Cardiol. 24 1082-1090.

Akselrod, S., Gordon, D., Ubel, F. A., Shannon, D. C., Barger, A. C., and Cohen, R. J. (1981). Power spectrum analysis of heart rate fluctuations: a quantitative probe of beat-to-beat cardiovascular control. Science 213, 220-222.

Angelone, A., and Coulter, N. A. (1964). Respiratory sinus arrhythmia: a frequency dependent phenomenon. J. Appl. Physiol. 19, 479-482.

Appel, M. L., Berger, R. D., Saul, J. P., Smith, J. M., and Cohen, R. J. (1989). Beat to beat variability in cardiovascular variables: noise or music? J. Am. Coll. Cardiol. 14, 1139-1148.
Bainbridge, F. A. (1930). The relation between respiration and the pulse-rate. J. Physiol. (Lond.) 54, 192-202.

Bernardi, L., Keller, F., Sanders, M., Reddy, P. S., Griffith, B., Meno, F., et al. (1989). Respiratory sinus arrhythmia in the denervated human heart. J. Appl. Physiol. 67, 1447-1455.

Berntson, G. G., Bigger, J. T., Eckberg, D. L., Grossman, P., Kaufmann, P. G., Malik, M., et al. (1997). Heart rate variability: origins, methods, and interpretive caveats. Pyschophysiology 34, 623-648.

Billman, G. E. (2009). Cardiac autonomic neural "remodeling" and susceptibility to sudden cardiac death: effect of endurance exercise training. Am. J. Physiol. Heart Circ. Physiol. 297, H1171-H1193.

Billman, G. E. (2011). Heart rate variability - a historical perspective. Front. Physio. 2:86. doi: 10.3389/fphys.2011.00086

Billman, G. E., and Dujardin, J.-P. (1990). Dynamic changes in cardiac vagal tone as measured by timeseries analysis. Am. J. Physiol. Heart Circ. Physiol. 258, H896-H902.

Billman, G. E., and Hoskins, R. S. (1989). Time-series analysis of heart rate variability during submaximal exercise. Evidence for reduced cardiac vagal tone in animals susceptible to ventricular fibrillation. Circulation 80, 146-157.

Billman, G. E., and Kukielka, M. (2007). Effect of endurance exercise training on the heart rate onset and heart rate recovery responses to submaximal exercise in animals susceptible to ventricular fibrillation. J. Appl. Physiol. 102, 231-240.

Brown, T. E., Beightol, L. A., Koh, J., and Eckberg, D. L. (1993). Important influence if respiration on human R-R interval power spectra is largely ignored. J. Appl. Physiol. 75, 2310-2317.

Chess, G. F., Tam, R. M. K., and Calaresu, F. R. (1975). Influence of cardiac neural inputs on rhythmic variations of heart period in the cat. Am. J. Physiol. 228, 775-780.

Cohen, M., and Taylor, J. A. (2002). Short-term cardiovascular oscillations in man: measuring and modeling the physiologies. J. Physiol. (Lond.) 542, 669-683.

Davies, C. T., and Neilson, J. M. M. (1967). Sinus arrhythmia in man at rest. J. Appl. Physiol. 22, 947-955.

Denver, J. W., Reed, S. F., and Porges, S. W. (2007). Methodological issues in the quantification of respiratory sinus arrhythmia. Biol. Psychol. 74, 286-294.

Eckberg, D. L. (1983). Human sinus arrhythmia as an index of vagal cardiac outflow. J. Appl. Physiol. 54, 961-966.

Eckberg, D. L. (1980). Nonlinearities in the human carotid baroreceptor reflex in human. Circ. Res. 47, 208-216.

Eckberg, D. L. (1997). Sympathovagal balance: a critical appraisal. Circulation 96, 3224-3232.

Eckberg, D. L., Mohanty, S. K., and Raczkowska, M. (1984). Trigeminal-baroreceptor reflex interactions modulate human cardiac avgal efferent activity. J. Physiol. (Lond.) 347, 75-83.

Fagius, J., and Sundlof, G. (1986). The diving response in man: effects on sympathetic activity in muscle and skin nerve fascicles. J. Physiol. (Lond.) 377, 429-443. 
Fleiss, J. L., Bigger, J. T., and Rolinitzky, L. M. (1992). The correlation between heart period variability and mean period length. Stat. Med. 11, 125-129.

Goldberger, J. J., Ahmed, M. W., Parker, M. A., and Kadish, A. H. (1994). Dissociation of heart rate variability from parasympathetic tone. Am. J. Physiol. Heart Circ. Physiol. 266, H2152-H2157.

Hainsworth, R. (1974). Circulatory responses from lung inflation in anesthetized dogs. Am. J. Physiol. $226,247-255$.

Hasking, G. J., Esler, M. D., Jennings, G. L., Burton, D., Johns, J. A., and Korner, P. I. (1986). Norepinephrine spillover to plasma in patients with congestive heart failure: evidence of increased overall and cardiorenal sympathetic nervous activity. Circulation 73, 615-621.

Hedman, A. E., Hartikainen, J. E., Tahvanainen, K. U., and Hakumaki, M. O. (1995). The high frequency component of heart rate variability reflects cardiac parasympathetic modulation rather than parasympathetic 'tone'. Acta Physiol. Scand. 155, 267-273.

Hirsch, J. A., and Bishop, B. (1981). Respiratory sinus arrhythmia in humans: how breathing pattern modulated heart rate. Am. J. Physiol. Heart Circ. Physiol. 241, H620-H629.

Hopf, H. B., Skyschally, A., Heusch, G., and Peters, J. (1995). Low-frequency spectral power of heart rate variability is not a specific marker of cardiac sympathetic modulation. Anesthesiology 82, 609-619.

Horner, S. M., Murphy, C. F., Coen, B., Dick, D. J., Harrison, F. G., Vespalcova, A., et al. (1996). Contributions of heart rate variability by mechanoelectric feedback. Stretch of the sinoatrial node reduced heart rate variability. Circulation 94, 1762-1767.

Houle, M. S., and Billman, G. E. (1999). Lowfrequency component of the heart rate variability spectrum: a poor marker of sympathetic activity. Am. J. Physiol. Heart Circ. Physiol. 267, $\mathrm{H} 215-\mathrm{H} 223$.

Jardine, D. L., Charles, C. J., Melton, I. C., May, C. N., Forrester, M. D., Frampton, C. M., et al. (2002). Continual recordings of cardiac sympathetic nerve activity in conscious sheep. Am. J. Physiol. Heart Circ. Physiol. 282, H93-H99.

Katona, P. G., Poitras, J. W., Barnett, G. O., and Terry, B. S. (1970). Cardiac vagal efferent and heart period in the carotid sinus reflex. Am. J. Physiol. 218, 1030-1037.

Kingwell, B. A., Thompson, J. M., Kaye, D. M., McPerherson, G. A., Jennings, G. L., and Esler, M. D. (1994). Heart rate spectral analysis, cardiac norepinephrine spillover, and muscle sympathetic nerve activity during human sympathetic nervous activation and failure. Circulation 90, 234-240.

Kleiger, R. E., Miller, J. P., Bigger, J. T. Jr., and Moss, A. J. (1987). Decreased heart rate variability and its association with increased mortality after acute myocardial infarction. Am. J. Cardiol. 59, 256-262.

Koh, J., Brown, T. E., Beightol, L. A., Ha, C. Y., and Eckberg, D. L. (1994). Human autonomic rhythms: vagal cardiac mechanisms in teraplegic subjects. J. Physiol. (Lond.) 474, 483-495.
Kollai, M., and Mizsei, G. (1990). Respiratory sinus arrhythmia is a limited measure of cardiac parasympathetic control in man. J. Physiol. (Lond.) $424,329-342$.

Levy, M. N. (1971). Sympathetic-parasympathetic interactions in the heart. Circ. Res. 29, 437-445.

Malliani, A., Pagani, M., Lombardi, F., and Cerutti, S. (1991). Cardiovascular neural regulation explored in the frequency domain. Circulation 84, 482-492.

Melcher, A. (1976). Respiratory sinus arrhythmia in man. A study in heart rate regulating mechanisms. Acta Physiol. Scand. Suppl. 435, 1-31.

Moak, J. P., Goldstein, D. S., Eldadah, B. A., Saleem, A., Holmes, C., Pechnik, S., et al. (2007). Supine low-frequency power of heart rate variability reflects baroreflex function, not cardiac sympathetic innervation. Heart Rhythm 4, 1523-1529.

Notarius, C. F., and Floras, J. S. (2001). Limitations of the use of spectral analysis of heart rate variability for the estimation of cardiac sympathetic activity in heart failure. Europace 3, 29-38.

Pagani, M., Lombardi, F., Guzzetti, S., Rimoldi, O. Furlan, R., Pizzinelli, P., et al. (1986). Power spectral analysis of heart rate and arterial pressure variabilities as a marker of sympatho-vagal interactions in man and conscious dog. Circ. Res. 59, 178-193.

Pagani, M., Lombardi, F., Guzzetti, S., Sandrone, G., Rimoldi, O., Malfatto, G., et al. (1984). Power spectral density of heart rate variability as an index of symptho-vagal interactions in normal and hypertensive subjects. J. Hypertens. Suppl. 2, 383-385.

Parati, G., di Rienzo, M., Castiglioni, P., Mancia, G., Taylor, J. A., and Studinger, P. (2006). Point:counterpoint: cardiovascular variability is/is not an index of autonomic control of circulation. J. Appl. Physiol. 101, 676-682.

Piccirillo, G., Ogawa, M., Song, J., Chong, V. J., Joung, B., Han, S., et al. (2009). Power spectral analysis of heart rate variability and autonomic nervous system activity measured directly in healthy dogs and dogs with tachycardia-induced heart failure. Heart Rhythm 6, 546-552.

Randall, D. C., Brown, D. R., Raisch, R. M., Yingling, J. D., and Randall, W. C. (1991). SA nodal parasympathectomy delineates autonomic control of heart rate power spectrum. Am. J. Physiol. Heart Circ. Physiol. 260, H985-H988.

Rea, R. F., and Eckberg, D. L. (1987). Carotid baroreceptor-muscle sympathetic relation in humans. Am. J. Physiol. 253, R929-R934.

Sacha, J., and Pluta, W. (2005). Different methods of heart rate variability analysis reveal different correlations of heart rate variability spectrum with average heart rate. J. Electrocardiol. 38 , $47-53$.

Sacha, J., and Pluta, W. (2008). Alterations of average heart rate change heart rate variability due to mathematical reasons. Int. J. Cardiol. 128 , 444-447.

Saul, J. P., Aria, Y., Berger, R. D., Lilly, L. S., Colucci, W. S., and Cohen, R. J. (1988). Assessment of autonomic regulation in chronic congestive heart failure by heart rate spectral analysis. Am. J. Cardiol. 61, 1292-1299.
Smith, L. L., Kukielka, M., and Billman, G. E. (2005). Heart rate recovery after exercise: a predictor of ventricular fibrillation susceptibility after myocardial infarction. Am. J. Physiol. Heart Circ. Physiol. 288, H1763-H1769.

Stramba-Badiale, M., Vanoli, E., De Farrari, G. M., Cerati, D., Foreman, R. D., and Schwartz, P. J. (1991). Sympathetic-parasympathetic interaction and accentuated antagonism in conscious dogs. Am. J. Physiol. Heart Circ. Physiol. 260, $\mathrm{H} 335-\mathrm{H} 340$.

Task Force of the European Society of Cardiology and the North American Society of Pacing and Electrophysiology. (1996). Heart rate variability: standards of measurement, physiological interpretation, and clinical use. Circulation 93, 1043-1065.

Taylor, J. A., Myers, C. W., Halliwill, J. R., Seidel, H., and Eckberg, D. L. (2001). Sympathetic restraint of respiratory sinus arrhythmia: implications for vagal-cardiac tone assessment in humans. Am. J. Physiol. Heart. Circ. Physiol. 280, H2804-H2814.

Thayler, J. F., Yamamoto, S. S., and Brosschot, J. F (2010). The relationship of autonomic imbalance, heart rate variability and cardiovascular disease risk factors. Int. J. Cardiol. 141, 122-131.

Uijtdehaage, S. H., and Thayer, J. F. (2000). Accentuated antagonism in the control of human heart rate. Clin. Auton. Res. 10, 107-110.

Van De Borne, P., Montano, N., Narkiewicz, K., Deguate, J. P., Mallani, A., Pagani, M., et al. (2001). Importance of ventilation in modulating interactions between sympathetic drive and cardiovascular variability. Am. J. Physiol. Heart Circ. Physiol. 280, H722-H729.

Van Hoogenhuyze, D., Weinstein, N., Martin, G. J., Weiss, J. S., Schaad, J. W., Sahyouni, X. N., et al. (1991). Reproducibility and relations to mean heart rate variability in normal subjects and patients with congestive heart failure secondary to coronary artery disease. Am. J. Cardiol. 68, 1668-1676.

Watson, A. M. D., Hood, S. G., Ramchandra, R., McAllen, R. M., and May, C. N. (2007). Increased cardiac sympathetic nerve activity in heart failure is not due desensitization of the arterial baroreflex. Am. J. Physiol. Heart Circ. Physiol. 293, H798-H804.

Received: 29 January 2013; accepted: 02 February 2013; published online: 20 February 2013.

Citation: Billman GE (2013) The LF/HF ratio does not accurately measure cardiac sympatho-vagal balance. Front. Physio. 4:26. doi: 10.3389/fphys.2013.00026

This article was submitted to Frontiers in Clinical and Translational Physiology, a specialty of Frontiers in Physiology.

Copyright () 2013 Billman. This is an open-access article distributed under the terms of the Creative Commons Attribution License, which permits use, distribution and reproduction in other forums, provided the original authors and source are credited and subject to any copyright notices concerning any third-party graphics etc. 